\title{
Asociación entre depresión y dependencia funcional en pacientes adultos mayores
}

Fernando M. Runzer-Colmenares ${ }^{1,2,3}$, Guida Castro ${ }^{1}$, Andrea Merino ${ }^{1}$, Cristina Torres-Mallma $^{3}$, Gregory Diaz ${ }^{1}$, Carolina Perez ${ }^{1}$, José F. Parodi ${ }^{1,3}$

\section{RESUMEN}

Objetivo: Determinar la asociación entre depresión y dependencia funcional en pacientes adultos mayores del Centro Geriátrico Naval.

Materiales y métodos: Estudio retrospectivo, transversal y analítico de un análisis secundario de base de datos, con un tamaño muestral por conveniencia de 625 adultos mayores.

Resultados: En el análisis de regresión lineal de predicción de dependencia funcional según índice de Barthel, se encontró que, por cada punto obtenido en el cuestionario de Yesavage, disminuye en 4,8 \pm 1.82 puntos el índice de Barthel y, por 5 puntos, disminuye $29.99 \pm 3.62$ puntos. En relación con el Índice de Lawton, se encontraron resultados significativos respecto a la edad, sexo, grado militar, rendimiento físico, fuerza de prensión y depresión.

Conclusiones: Se evidenció asociación entre depresión y dependencia funcional para actividades básicas e instrumentales de la vida diaria. Asimismo, que a mayor riesgo de depresión, mayor probabilidad de presentar dependencia funcional según el índice de Barthel, independientemente de otras variables.

Palabras Claves: Adulto mayor; depresión; discapacidad (Fuente: DeSC BIREME).

\section{Association between depression and functional dependence in elderly patients}

\section{ABSTRACT}

Objective: To determine the association between depression and functional dependence in elderly patients of the Centro Geriátrico Naval (Peruvian naval medical center for elderly people).

Materials and methods: A retrospective, cross-sectional, analytic study conducted from a secondary data analysis, with a convenience sample size of 625 elderly people.

Results: In the linear regression analysis which predicts functional dependence according to the Barthel Index, it was found that, for each point in the Yesavage questionnaire, the Barthel Index score decreases $4.8 \pm 1.82$ points and, for every 5 points, it decreases $29.99 \pm 3.62$ points. In relation to the Lawton Index, significant outcomes were found regarding age, gender, military rank, physical performance, grip strength and depression.

Conclusions: The results show an association between depression and functional dependence in performing basic and instrumental activities of daily life. Moreover, the higher the risk of depression, the higher the chance of developing functional dependence according to the Barthel Index, regardless of other variables.

Keywords: Elderly; depression; disability (Source: MeSH NLM).

1. Centro de Investigación del Envejecimiento (CIEN).

2. Servicio de Geriatría, Centro Médico Naval. Callao, Perú.

3. Facultad de Medicina Humana, Universidad de San Martín de Porres. Lima, Perú 
Fernando M. Runzer-Colmenares, Guida Castro, Andrea Merino, Cristina Torres-Mallma, Gregory Diaz, Carolina Perez, José F. Parodi

\section{INTRODUCCIÓN}

La depresión es una enfermedad muy común en la población general y muy presente en la población adulta mayor, con una prevalencia en el Perú del $23 \%{ }^{(1)}$. A nivel hospitalario local, la prevalencia es mayor, llegando al $50 \%{ }^{(2)}$. Por ello la depresión se considera como un problema frecuente de salud y carga de enfermedad ${ }^{(3,4)}$.

En geriatría, se utilizan diversos instrumentos para valorar los síntomas depresivos como la escala de Hamilton, Montgomery-Asberg Depression Rating Scale (MADRS), Self-Rating Depression Scale (SDS) de Zung, Beck Depression Inventory (BDI), Minnesota Multiphasic Personality Inventory (MMPI), Symptom Checklist-90Revised, entre otros. La Escala de Depresión Geriátrica (Geriatric Depression Scale - GDS) de Yesavage cuenta con diferentes versiones, siendo las mayormente utilizadas las de 30, 15 y 5 ítems, con una sensibilidad mayor del $80 \%$ y una especificidad mayor del $70 \%$. La versión de 5 ítems está validada para varones con alta sensibilidad y valor predictivo negativo ${ }^{(5,6)}$ y es ampliamente utilizada en nuestra región ${ }^{(2,7)}$. Existe evidencia de que la depresión, medida con este instrumento, logra una correcta evaluación de síntomas afectivos y, adicionalmente, ayuda a la evaluación integral del adulto mayor en proceso de rehabilitación, en especial en la valoración funcional ${ }^{(8)}$.

Por otro lado, la funcionalidad se define como la capacidad del individuo para llevar a cabo de una forma efectiva las actividades de la vida diaria ${ }^{(9,10)}$. La dependencia funcional es un problema importante de salud pública por estar estrechamente relacionado con mortalidad, discapacidad, enfermedades crónicas, enfermedades mentales, problemas sociales y pobre calidad de vida ${ }^{(11-14)}$.

Diversos autores han encontrado una relación importante entre depresión y riesgo de dependencia funcional ${ }^{(13,15-19)}$, inclusive se conoce que ésta aporta a modelos predictivos de trastornos depresivos de evolución tórpida ${ }^{(4,15)}$. Adicionalmente, la posibilidad de presentar dependencia funcional se duplica en pacientes con síntomas depresivos ${ }^{(18)}$.

No obstante, es controversial el rol de las comorbilidades en la asociación entre estas variables, por ello es importante valorar la relación que hay entre depresión y dependencia funcional en nuestra región y si hay influencia de otros factores. Así mismo, existe controversia en la literatura con respecto a la asociación de dependencia funcional para actividades instrumentales de la vida diaria (AIVD) y depresión ${ }^{(20)}$; por lo que el objetivo del presente estudio es cuantificar la asociación entre las medida con los índices de Barthel y Lawton y depresión en adultos mayores.

\section{MATERIALES Y MÉTODOS}

\section{Diseño del estudio}

El presente es un estudio retrospectivo, transversal, analítico, que se realizó mediante un análisis secundario de datos del estudio "Asociación entre dependencia funcional y fragilidad en adultos mayores", que contó con la participación de 625 pacientes. El tamaño muestral fue de 574 adultos mayores, pues se excluyeron a los datos de los participantes con un puntaje de Minimental State Examination menor a 21 (51 participantes).

\section{Procedimiento del estudio}

La ficha de recolección de datos incluyó información sociodemográfica, de valoración funcional y afectiva. Para mayor información sobre la recolección de datos y características de las variables, revisar el artículo original ${ }^{(21)}$.

\section{Medición de las variables:}

\section{Socio-demográfico}

Se constató el género de cada participante y la edad como variable numérica. Además, el grado militar se categorizó en: civil, personal subalterno y personal superior. Se consignó la variable "Vivir sólo", considerándose positiva al responder afirmativamente el paciente a la pregunta “¿Vive sólo?”.

\section{Valoración Funcional}

Dependencia funcional para actividades básicas de la vida diaria (ABVD): según índice de Barthel, que consigna: alimentación, aseo, capacidad de vestirse, continencia, uso de sanitario, transferencia y uso de escaleras. A mayor puntaje, mejor desempeño de ABVD (0-100). Para el análisis, se decidió dicotomizar la variable de tal modo que sólo a los participantes con puntaje de 100 se les consideró como independientes para ABVD.

Dependencia funcional para AIVD: según índice de Lawton, que consigna uso de teléfono, compras, manejo de fármacos, preparación de alimentos, cuidado de casa, lavado de ropa, uso de medios de transporte y uso de dinero. A mayor puntaje, mejor desempeño de AIVD (0-8). Para el análisis se categorizó en tres grupos: Puntaje de 0-3 (dependencia total-severa), puntaje de 4-6 (dependencia moderada) y puntaje de 7-8 (leve-independencia). ${ }^{(22,23)}$

Rendimiento físico, según la batería corta de rendimiento físico (Short physical performance Battery - SPPB), que consigna evaluación de equilibrio, de la marcha y de capacidad de levantarse/sentarse de una silla. A mayor puntaje, mejor desempeño físico (0-12). Para el análisis, se consideró a la variable como numérica.

Fuerza muscular, medida con un dinamómetro manual (MODEL Dynamometer, series 120286). Se solicitó al participante a tomar asiento y se procedió a medir la fuerza de agarre en mano dominante con brazo extendido hacia abajo, en dos oportunidades, tomando el mejor valor. Expresada en kg e incluida en el análisis como numérica.

\section{Valoración afectiva}

Se utilizó el Cuestionario de Yesavage de 5 ítems, recomendado por el Ministerio de Salud del Perú según la Valoración Clínica del Adulto Mayor (VACAM). Se constató el puntaje global y se tomó como numérica. Las preguntas 
que conforman al cuestionario son: ¿Está básicamente satisfecho con su vida?, ¿Se siente a menudo aburrido?, ¿Se siente a menudo sin esperanza?, ¿Prefiere quedarse en casa más que salir a hacer cosas nuevas? y ¿Piensa que no vale para nada tal como está ahora?.

\section{Recolección y análisis de datos}

Respecto a la recolección de datos, se utilizó el Software Microsoft Excel para elaborar tablas dinámicas, en donde se recolectó la información de la base de datos del estudio principal. El análisis se realizó utilizando el programa estadístico Stata versión 13.0, considerando un nivel de significancia de $p<0.05$.

ra el análisis de variables categóricas se utilizó la prueba de chi cuadrado, T de student para análisis de variables numéricas de distribución normal y test de Kruskall Wallis y ANOVA oneway en caso de variables de tres o más categorías. Además se realizaron dos análisis bivariados en relación a dependencia funcional según índice de Barthel y Lawton, además de la construcción de un modelo de regresión lineal múltiple para predicción de incremento o descenso en el puntaje del índice de Barthel, en base a depresión.

\section{Aspectos éticos}

El estudio original y el actual contaron con la autorización del Comité de Ética del Centro Médico Naval del Perú, toda la información relacionada al mismo, fue archivada de manera segura en el centro de investigación. La información de los pacientes esta almacenada de manera electrónica protegidas con contraseñas, que solo las manejan el personal involucrado en el estudio y serán de acceso limitado. En este estudio no se cuenta con ninguna documentación de los participantes del estudio original. Por otro lado, la información contenida en la base de datos no contiene nombre, ni dirección de los participantes.

\section{RESULTADOS}

Encontramos asociación entre el score del cuestionario de Yesavage y dependencia funcional, tanto para ABVD como para AIVD, existiendo una relación inversamente proporcional entre ambos puntajes y depresión.

Encontramos una media de edad de 77.4 + 8.6 años, $58.01 \%$ fueron de sexo masculino y la mayoría personal militar subalterno (51.09\%). La prevalencia de dependencia funcional para ABVD según el Índice de Barthel fue de $37.57 \%$ y para AIVD según Índice de Lawton de $49.5 \%$. Al evaluar la variable SPPB, se obtuvo una media de $8.6 \pm$ 3.5 puntos y para fuerza de prensión de $28.7 \pm 12.2 \mathrm{~kg}$. El promedio del puntaje del Cuestionario de Yesavage fue de $1.5 \pm 1.5$ puntos, adicionalmente, el $43.95 \%$ de participantes tuvo un tamizaje positivo para depresión (>2 puntos) (Tabla 1$)$.

Tabla 1. Características de los participantes del estudio $(n=574)$

\begin{tabular}{|c|c|c|c|}
\hline Variables & $\mathrm{n}$ & $\%$ & media $\pm D^{1}$ \\
\hline Edad & & & $77.4 \pm 8.6$ \\
\hline \multicolumn{4}{|l|}{ Sexo } \\
\hline Masculino & 333 & 58.01 & \\
\hline Femenino & 241 & 41.99 & \\
\hline \multicolumn{4}{|l|}{ Grado Militar } \\
\hline Subalterno & 257 & 51.09 & \\
\hline Superior & 66 & 13.12 & \\
\hline Civil & 180 & 35.79 & \\
\hline Vive solo & 65 & 11.38 & \\
\hline \multicolumn{4}{|l|}{ Dependencia Funcional } \\
\hline Barthel 0-90 puntos & 210 & 37.57 & \\
\hline Barthel $95-100$ puntos & 349 & 62.43 & \\
\hline $\begin{array}{l}\text { Actividades Instrumentales } \\
\text { (Lawton) }\end{array}$ & 199 & 64 & \\
\hline 0 a 3 puntos & 149 & 29.5 & \\
\hline 4 a 6 puntos & 250 & 49.5 & \\
\hline 7 a 8 puntos & 106 & 20.99 & \\
\hline $\mathrm{SPPB}^{2}$ & & & $8.6 \pm 3.5$ \\
\hline Fuerza de Prensión en kg & & & $28.7 \pm 12.2$ \\
\hline Cuestionario de Yesavage & 57 & 18.3 & \\
\hline 0 puntos & 153 & 32.48 & \\
\hline 1 punto & 111 & 23.57 & \\
\hline 2 puntos & 104 & 22.08 & \\
\hline 3 puntos & 42 & 8.92 & \\
\hline
\end{tabular}




\begin{tabular}{|c|c|c|}
\hline 4 puntos & 36 & 7.64 \\
\hline 5 puntos & 25 & 5.31 \\
\hline $\begin{array}{l}\text { Depresión (Yesavage de } 5 \\
\text { ítems) } \\
1 \text { DE: Desviación Estándar }\end{array}$ & & $1.5 \pm 1.5$ \\
\hline
\end{tabular}

1 DE: Desviación Estándar

2 Short Physical Performance Battery

En el análisis bivariado de dependencia funcional según índice de Barthel, se encontró asociación estadísticamente significativa con el grado militar, encontrando menor prevalencia de dependencia para ABVD en el personal civil. Asimismo, se encontró una alta frecuencia de autoreporte de vivir solo en el grupo con dependencia funcional, así como promedios significativamente mayores de puntaje de
SPPB y fuerza de prensión en el grupo con independencia funcional, sin encontrar diferencias para las variables sexo y edad. Finalmente, obtuvimos promedios mayores del puntaje del cuestionario de Yesavage en el grupo con dependencia funcional, con resultados estadísticamente significativos (Tabla 2).

Tabla 2. Análisis bivariado de dependencia funcional según indice de Barthel $(n=574)$

\begin{tabular}{|c|c|c|c|}
\hline Variables & $\begin{array}{c}\text { Dependencia para } \\
\text { ABVD }\end{array}$ & $\begin{array}{c}\text { Independencia } \\
\text { ABVD }\end{array}$ & Valor de $\mathrm{P}$ \\
\hline Edad (media $\pm \mathrm{DE}^{1}$ ) & $76.71 \pm 10.12$ & $77.52 \pm 7.59$ & $0.3^{2}$ \\
\hline Sexo n (\%) & & & $0.9^{3}$ \\
\hline Masculino & $120(37.62)$ & 199 (62.38) & \\
\hline Femenino & $90(37.97)$ & $147(62.03)$ & \\
\hline Grado Militar n (\%) & & & $0.022^{3}$ \\
\hline Subalterno & $95(38.15)$ & $154(61.85)$ & \\
\hline Superior & $30(45.45)$ & $36(54.55)$ & \\
\hline Civil & $51(28.33)$ & 129 (71.67) & \\
\hline Vive solo n (\%) & $10(15.38)$ & 55 (84.62) & $<0.0001^{3}$ \\
\hline $\mathrm{SPPB}^{5}\left(\right.$ media $\left.\pm \mathrm{DE}^{1}\right)$ & $7.26 \pm 4.15$ & $9.40 \pm 2.83$ & $<0.0001^{2}$ \\
\hline $\begin{array}{l}\text { Fuerza de Prensión en kg } \\
\text { (media } \pm D^{1} \text { ) }\end{array}$ & $26.53 \pm 14.09$ & $30.07 \pm 11.0$ & $0.002^{2}$ \\
\hline $\begin{array}{l}\text { Cuestionario de Yesavage } \\
\left(\text { media } \pm D^{1}\right)\end{array}$ & $1.75 \pm 1.47$ & $1.40 \pm 1.48$ & $0.004^{6}$ \\
\hline \multicolumn{4}{|c|}{$\begin{array}{ll}1 & \text { DE: Desviación Estándar } \\
2 & \text { T de Student } \\
3 & \text { Prueba de chi² } \\
4 & \text { Actividades básicas de la vida diaria (índice de Barthel) } \\
5 & \text { Short Physical Performance Battery } \\
6 & \text { Kruskall Wallis }\end{array}$} \\
\hline
\end{tabular}

En el análisis bivariado de dependencia para AIVD, encontramos asociación significativa entre edad y severidad de dependencia funcional. Se evidenció mayor dependencia en el género masculino y en personal militar. Además, obtuvimos promedios de puntaje de SPPB y fuerza de prensión significativamente menores en el grupo con dependencia total-severa. No encontramos significancia estadística con la variable "vivir solo". Finalmente, existe una asociación estadística entre depresión y AIVD, con promedios mayores del score del Cuestionario de Yesavage en el grupo con dependencia total-severa (Tabla 3). 
Tabla 3. Análisis bivariado de dependencia para AIVD ${ }^{4}$ según índice de Lawton $(n=574)$

\begin{tabular}{|c|c|c|c|c|}
\hline Variables & $\begin{array}{c}\text { Dependencia } \\
\text { Severa-Total (Lawton } \\
0-3 \text { puntos) }\end{array}$ & $\begin{array}{l}\text { Dependencia } \\
\text { Moderada (Lawton } \\
\text { 4-6 puntos) }\end{array}$ & $\begin{array}{l}\text { Dependencia Leve- } \\
\text { Independencia } \\
\text { (Lawton 7-8 puntos) }\end{array}$ & Valor de $p$ \\
\hline Edad (media $\pm \mathrm{DE}^{1}$ ) & $80.39 \pm 8.46$ & $76.51 \pm 8.80$ & $73.68 \pm 7.13$ & $0.007^{2}$ \\
\hline Sexo n (\%) & & & & $0.001^{3}$ \\
\hline Masculino & $87(30.74)$ & $155(54.77)$ & $41(14.49)$ & \\
\hline Femenino & $62(28.31)$ & $95(43.38)$ & $62(28.31)$ & \\
\hline Grado Militar n (\%) & & & & $<0.0001^{3}$ \\
\hline Subalterno & $63(27.39)$ & $140(60.87)$ & $27(11.74)$ & \\
\hline Superior & 20 (35.09) & $17(29.82)$ & 20 (35.09) & \\
\hline Civil & $44(25.0)$ & $89(50.57)$ & $43(24.43)$ & \\
\hline Vive solo $\mathrm{n}(\%)$ & $15(24.59)$ & $36(59.02)$ & $10(21.07)$ & $0.2^{3}$ \\
\hline $\operatorname{SPPB}^{5}\left(\right.$ media $\left.\pm \mathrm{DE}^{1}\right)$ & $5.46 \pm 3.5$ & $10.29 \pm 2.04$ & $10.04 \pm 2.29$ & $<0.0001^{2}$ \\
\hline $\begin{array}{l}\text { Fuerza de Prensión en kg } \\
\left.\text { (media } \pm D E^{1}\right)\end{array}$ & $19.55 \pm 7.73$ & $34.31 \pm 10.92$ & $30.45 \pm 11.9$ & $0.029^{2}$ \\
\hline $\begin{array}{l}\text { Cuestionario de Yesavage } \\
\left.\text { (media } \pm D^{1}\right)\end{array}$ & $2.96 \pm 1.55$ & $0.90 \pm 0.95$ & $1.13 \pm 1.20$ & $<0.0001^{2}$ \\
\hline
\end{tabular}

1 DE: Desviación Estándar

2 ANOVA (oneway)

3 Prueba de $\mathrm{chi}^{2}$

4 Actividades Instrumentales de la Vida Diaria

5 Short Physical Performance Battery

Respecto al análisis de regresión lineal de predicción de dependencia funcional, se incluyó como variable "desenlace" al Índice de Barthel como valor numérico. Encontramos resultados estadísticamente significativos con las variables edad, sexo, SPPB, fuerza de prensión, AIVD y depresión, tanto de manera independiente como en el modelo global (Tabla 4).

Tabla 4. Regresión lineal de predicción de dependencia funcional $(n=574)$

$\begin{array}{lccc}\text { Variables } & \text { Coeficiente }^{1} & \text { Error Estándar } & \text { Valor de } \mathbf{p} \\ \text { Cuestionario de Yesavage } & & & 0.006 \\ \quad \text { 1 Punto } & -4.8 & 1.82 & 0.04 \\ \mathbf{2} \text { Puntos } & -7.5 & 1.83 & 0.001 \\ \mathbf{3} \text { Puntos } & -11.9 & 2.57 & 0.05 \\ \mathbf{4} \text { Puntos } & -16.14 & 3.23 & 0.001 \\ \mathbf{5} \text { Puntos } & -29.99 & 3.62 & <0.0001 \\ \text { Edad } & -0.31 & 0.8 & <0.0001 \\ \text { Índice de Lawton } & 15.1 & 4.03 & 0.018 \\ \text { SPPB } & 21.78 & 0.08 & 0.002 \\ \text { Fuerza de Prensión } & 3.08 & 1.08 & <0.0001 \\ \text { Modelo Total }^{2} & & & \end{array}$

1 Coeficiente de Regresión Lineal de predicción de Dependencia Funcional según Índice de Barthel ajustado por: valores de cuestionario de depresión de Yesavage,

Edad, AIVD, Short Physical Performance Battery y Fuerza de Prensión.

2 Prob $>F$ 
Fernando M. Runzer-Colmenares, Guida Castro, Andrea Merino, Cristina Torres-Mallma, Gregory Diaz, Carolina Perez, José F. Parodi

\section{DISCUSIÓN}

El presente estudio tuvo como objetivo demostrar y cuantificar la asociación entre dependencia funcional y depresión en adultos mayores. Encontramos que los participantes con puntajes altos en el Cuestionario de Yesavage presentaron mayor dependencia funcional según el índice de Barthel. Dichos resultados se asociaron estadísticamente con las variables: edad, fuerza de prensión y puntajes de índice de Lawton y SPPB.

Existe evidencia acerca del rol de los síntomas depresivos en torno a la independencia funcional en ancianos, donde el Cuestionario de Yesavage logra predecir una evolución desfavorable en la recuperación funcional de pacientes con enfermedad cardiovascular, tanto para ABVD como para AIVD ${ }^{(22)}$. Adicionalmente, existe escasa evidencia en relación a la asociación entre depresión y dependencia funcional medidas con el Cuestionario de Yesavage e índice de Barthel, respectivamente, habiéndose encontrado mayor información con otros instrumentos, como el índice de Katz, que logra asociar al pobre desempeño en ABVD como factor de riesgo para depresión en ancianos ${ }^{(24)}$.

Por otro lado, existe evidencia respecto a la importancia de la actividad física y mejoría de la funcionalidad en trastornos depresivos en adultos mayores, sin embargo, dicha bibliografía no utiliza el índice de Barthel como herramienta de valoración funcional ${ }^{(25,26)}$.

Asimismo, se encontró que en el personal militar en situación de retiro obtuvo mayor frecuencia de dependencia para ABVD y AIVD. En relación a ello, un estudio en diabéticos militares retirados, concluye que la dependencia funcional es mucho más frecuente que en la población general ${ }^{(27)}$. No obstante, otro estudio que menciona motivos de consultas a centros médicos militares, no evidencian diferencias al respecto entre personal civil y militar ${ }^{(28)}$.

En cuanto a la asociación entre depresión y dependencia funcional, ha sido estudiada por diversos autores como Ávila-Funes, Patiño, Ayan, García-Peña, Barrantes con resultados variables según los instrumentos de medición y la población diana ${ }^{(4,13,15-17,29,30)}$. Nuestro principal hallazgo, similar a los estudios revisados, es una fuerte asociación entre depresión y dependencia funcional, hallando una relación inversamente proporcional entre cuestionario de Yesavage y el índice de Barthel, independiente de la edad, índice de Lawton, SPPB y fuerza de prensión.

En cuanto a la dependencia funcional según índice de Barthel se encontró diferencias estadísticamente significativas $(p=0.022)$ según grado militar, donde se observó que la mejor performance fue para el personal civil (71.67\% independientes para ABVD) y la peor performance fue para el personal oficial $(45.45 \%$ dependientes para
$A B V D)$. Además, al comparar grado militar y dependencia para actividades instrumentales de la vida diaria, encontramos que el mayor porcentaje para dependencia severa lo obtuvo el grupo de oficiales (35.09\%).

Si bien se esperaría encontrar un mayor grado de dependencia en personal subalterno en comparación con los oficiales, el presente estudio evidencia lo contrario; probablemente explicados por la influencia de factores que no han sido contemplados en este estudio (uso de otros servicios de salud por parte del personal militar superior, por ejemplo), por lo que se necesitan estudios posteriores para esclarecer estos hallazgos.

En cuanto a si el paciente vive o no solo, encontramos que el mayor porcentaje de los que son independientes para $A B V D$, viven solos $(84.62 \%, p<0.0001)$, lo que concuerda con lo esperado ya que, para poder vivir solo, se debe contar con la posibilidad de auto asistencia mínima, resultado similar en estudios previamente realizados ${ }^{(1,11,31}$ ${ }^{33)}$. Sin embargo, no deja de ser un factor de riesgo para complicaciones físicas y afectivas a largo plazo ${ }^{(34,35)}$.

En relación a rendimiento físico, se encontró una relación directamente proporcional a la independencia para ABVD y para AIVD lo que también es un resultado esperado ya que, a mayor puntaje en el SPPB, se entiende que desarrolla mejor la prueba, lo que garantiza su autosuficiencia. Con respecto a fuerza de prensión, mientras mayor sea esta, se obtiene mayor puntaje en el índice de Barthel, resultado que se encuentra dentro de lo esperado, ya que la fuerza de presión se correlaciona con la fuerza del muslo ${ }^{(36)}$.

El índice de Lawton presenta ciertas diferencias en cuanto a puntaje según sexo, pues nuestra realidad cultural limita a las mujeres adultas mayores a realizar más tareas del hogar que sus esposos. Estas variaciones sociodemográficas son las mismas encontradas en estudios anteriores y dependen de la población, muestra y métodos utilizados ${ }^{(3,32,37)}$. Además. debemos tener en cuenta que las actividades entre hombres y mujeres suelen diferir en todas las etapas de la vida ${ }^{(2,9,10,38-41)}$. Sin embargo, resulta útil el empleo del índice de Lawton, ya que según algunos autores, puede detectar de manera precoz una dependencia funcional ${ }^{(42,43)}$.

Nuestro estudio tiene algunas limitaciones: Por haber realizado el estudio en una población militar retirada y en sus familiares, sus resultados pueden ser difíciles de extrapolar a la población general. Al ser vistos los pacientes en un centro especializado, probablemente se haya incurrido en sesgo hospitalario. Por ser nuestra metodología de corte transversal no se podrán estimar riesgos. Si bien los resultados no pueden ser extrapolables por presentar sesgo hospitalario, cabe resaltar que 
la Marina de Guerra del Perú no cuenta con atención primaria, por ello los participantes incluidos en el presente estudios no cumplían criterios de evaluación especializada por sus comorbilidades y no necesariamente todos los participantes son complejos. Asimismo, el presente estudio no tuvo como objetivo determinar riesgos, pero el análisis de regresión lineal múltiple nos da una aproximación por cuantificación de asociación entre variable dependiente e independiente.

En conclusión, existe una asociación fuerte e independiente entre depresión y dependencia funcional.

Además, es de gran importancia clínica cuantificar de manera aproximada cuánto podría disminuir el puntaje en el índice de Barthel por cada punto en el cuestionario de Yesavage y determinar que la sola intervención en los síntomas depresivos en el adulto mayor contribuiría a mejorar su estado funcional y con ello la calidad de vida ${ }^{(12)}$. De esta manera, se sugiere aplicar la valoración geriátrica integral, que incluye la valoración cognitivo-afectiva y de desempeño funcional, en toda evaluación al adulto mayor, y proponer nuevos modelos de manejo integral y a tiempo de los síntomas depresivos y asimismo, poder contribuir al establecimiento de políticas de salud pública basadas en evidencia para fomentar su tratamiento temprano y prevención de la depresión.

\section{REFERENCIAS BIBLIOGRÁFICAS}

1. Guerra M, Ferri CP, Sosa AL, Salas A, Gaona C, Gonzales V, et al. Late-life depression in Peru, México and Venezuela: The 10/66 population-based study. Br J Psychiatry. 2009;195(6):510-5.

2. Rebagliati Tirado C, Runzer Colmenares F, Horruitiniere Izquierdo M, Lavaggi Jacobs G, Parodi García F. Caracterización clínica, epidemiológica y social de una población geriátrica hospitalizada en un Centro Geriátrico Militar. Horiz Med (Barcelona). 2012;12(2):21-7.

3. Colón-Emeric CS, Whitson HE, Pavon J, Hoenig H. Functional decline in older adults. Am Fam Physician. 2013;88(6):388-94.

4. Conradsson M, Littbrand H, Boström G, Lindelöf N, Gustafson Y, Rosendahl E. Is a change in functional capacity or dependency in activities of daily living associated with a change in mental health among older people living in residential care facilities? Clin Interv Aging. 2013;8:1561-8.

5. Abizanda P, Rodríguez L. Tratado de medicina geriátrica. Fundamentos de la atención sanitaria a los mayores. Elsevier; 2015

6. De Dios-del Valle R, Hernández-Sánchez A, Rexach- Cano L, Cruz-Jentoft A. Validación de una versión de cinco ítems de la Escala de Depresión Geriátrica de Yesavage en población española. Rev Esp Geriatr Gerontol [Internet]. 2001 [acceso en mayo de 2017];36(5):276-80. Disponible en: http://www. sciencedirect.com/science/article/pii/S0211139X01747361

7. Juárez J, León A, Linares VA. Evaluación del grado de depresión de adultos mayores de 60 años del AA. HH "Viña alta"-La Molina, Lima-Perú. Horiz Med (Barcelona). 2012;12(2):26-9.

8. Shahab S, Nicolici DF, Tang A, Katz P, Mah L. Depression Predicts Functional Outcome in Geriatric Inpatient Rehabilitation. Arch Phys Med Rehabil [Internet]. 2017 [acceso en mayo de 2017];98(3):500-7. Disponible en: http://dx.doi. org/10.1016/j.apmr.2016.07.014
9. Stessman J, Rottenberg Y, Shimshilashvili I, Ein-Mor E, Jacobs JM. Loneliness, health, and longevity. Journals Gerontol - Ser A Biol Sci Med Sci. 2014;69(6):744-50.

10. Rajan KB, Barnes LL, Wilson RS, Evans DA, Mendes de Leon CF. Racial Differences on Association of Depressive Symptoms With Combined Basic and Instrumental Activities of Daily Living. Journals Gerontol Ser A Biol Sci Med Sci [Internet]. 2014;69A(2):215-22. Available from: https://academic. oup.com/biomedgerontology/article-lookup/doi/10.1093/ gerona/glt074

11. Mendes De Leon CF, Rajan KB. Psychosocial influences in onset and progression of late life disability. Journals Gerontol - Ser B Psychol Sci Soc Sci. 2014;69(2):287-302.

12. Kwak M, Ingersoll-Dayton B, Burgard S. Receipt of care and depressive symptoms in later life: The importance of selfperceptions of aging. Journals Gerontol - Ser B Psychol Sci Soc Sci. 2014;69(2):325-35.

13. Ayan C, Cancela JM, Gutiérrez A, Prieto I. Influence of the cognitive impairment level on the performance of the Timed " Up \& Go" Test (TUG) in elderly institutionalized people. Arch Gerontol Geriatr. 2013;56(1):44-9.

14. Soyuer F, Senol V. Functional outcome and depression in the elderly with or without fatigue. Arch Gerontol Geriatr. 2011;53(2):164-7.

15. Villada P, Alonso F, Vélez A, Fernando E, Zuleta L. Physical Exercise and Depression in the Elderly: A Systematic Review. Rev Colomb Psiquiat. 2013; 42(2): 198-211.

16. Ávila-Funes JA, Melano-Carranza E, Payette H, Amieva H. Síntomas depresivos como factor de riesgo de dependencia en adultos mayores. Salud Publica Mex. 2007;49(5):367-75.

17. Gastelurrutia P, Lupón J, Altimir S, De Antonio M, González $B$, Cabanes R, et al. Effect of fragility on quality of life in patients with heart failure. Am J Cardiol. 2013 [acceso en mayo de 2017];112(11):1785-9. Disponible en: http://dx.doi. org/10.1016/j.amjcard.2013.08.002

18. Arias-Merino ED, Mendoza-Ruvalcaba NM, Ortiz GG, VelázquezBrizuela IE, Meda-Lara RM, Cueva-Contreras J. Physical function and associated factors in community-dwelling elderly people in Jalisco, Mexico. Arch Gerontol Geriatr. 2012;54(3).

19. Helvik A-S, Selbæk G, Engedal K. Functional decline in older adults one year after hospitalization. Arch Gerontol Geriatr. 2013 [acceso en mayo de 2017] ;57(3):305-10. Disponible en: http://linkinghub.elsevier.com/retrieve/pii/ S0167494313000964

20. Beekman AT, Deeg DJ, Braam AW, Smit JH, Van Tilburg W. Consequences of major and minor depression in later life: a study of disability, well-being and service utilization. Psychol Med. 1997;27(6):1397-409.

21. Runzer-Colmenares FM, Samper-Ternent $R$, Snih Al, Ottenbacher KJ, Parodi JF, Wong R, et al. Prevalence and factors Associated with Frailty Among Peruavian Older Adults. Arch Gerontol Geriatr. 2014;58(1):1-11.

22. Millan-Calenti JC, Tubio J, Pita-Fernandez S, GonzalezAbraldes I, Lorenzo T, Fernandez-Arruty T, et al. Prevalence of functional disability in activities of daily living (ADL), instrumental activities of daily living (IADL) and associated factors, as predictors of morbidity and mortality. Arch Gerontol Geriatr. 2010;50(3):306-10.

23. Camps $E$, Andreu L, Colomer M, Claramunt L, Pasaron $M$. Valoración del grado de autonomía funcional de pacientes renales crónicos según índices de Barthel, Lawton y baremo de Ley de Dependencia. Rev la Soc Española Enfermería Nefrológica. 2009 [acceso en mayo de 2017];12(2):28-34. Disponible en: http://scielo.isciii.es/scielo.php?pid=S1139$13752009000200005 \&$ script=sci_arttext\&tlng=e\%5Cnhttp: / / scielo.isciii.es/pdf/nefro/v12n2/original4.pdf 
24. Klein D, Pendergrass A, Becker C, Hautzinger M, Pfeiffer K. Dementia Caregiver Interventions: A Systematic Review of Care Recipient Outcome Measures. Int J Emerg Ment Heal Hum Resil. 2015;17(2):415-26.

25. Lund R, Nilsson CJ, Avlund K. Can the higher risk of disability onset among older people who live alone be alleviated by strong social relations? A longitudinal study of non-disabled men and women. Age Ageing. 2010;39(3):319-26.

26. Lai SM, Duncan PW, Keighley J, Johnson D. Depressive symptoms and independence in BADL and IADL. J Rehabil Res Dev. 2002;39(5):589-96.

27. Ahroni JH, Boyko EJ, Davignon DR, Pecoraro RE. The health and functional status of veterans with diabetes. Diabetes Care. 1994;17(4):318-21.

28. Kroenke K, Jackson JL. Outcome in general medical patients presenting with common symptoms: a prospective study with a 2-week and a 3-month follow-up. [Internet].

29. Barrantes-Monge M, García-Mayo EJ, Gutiérrez-Robledo LM, Miguel-Jaimes A. Dependencia funcional y enfermedades cr??nicas en ancianos mexicanos. Salud Publica Mex. 2007;49(SUPPL. 4):459-66.

30. Alexandre T da S, Corona LP, Nunes DP, Santos JLF, Duarte YA de 0 , Lebrão ML. Gender differences in incidence and determinants of disability in activities of daily living among elderly individuals: SABE study. Arch Gerontol Geriatr. 2012;55(2):431-7.

31. Dorantes-Mendoza G, Ávila-Funes J, Mejía-Arango S, GutiérrezRobledo L. Factores asociados con la dependencia funcional en los adultos mayores: un analisis secundario del Estudio Nacional sobre Salud y Envejecimiento en Mexico,2001. Rev Panam Salud Publica. 2007;22(1):1-11.

32. García-Peña C, Wagner FA, Sánchez-Garcí S, Espinel-Bermúdez $C$, Juárez-Cedillo T, Pérez-Zepeda $M$, et al. Late-life depressive symptoms: Prediction models of change. J Affect Disord [Internet]. 2013 [acceso mayo del 2017];150(3):886-94. Disponible en: http://dx.doi.org/10.1016/j.jad.2013.05.007

33. Fuentes-García A, Sánchez H, Lera L, Cea X, Albala C. Original: Desigualdades socioeconómicas en el proceso de discapacidad en una cohorte de adultos mayores de Santiago de Chile. Gac Sanit [Internet]. 2013 [acceso mayo del 2017];27(3):226-32. Disponible en: http://search.ebscohost.com/login.aspx?direc $\mathrm{t}=$ true\&db=edselp\&AN=S0213911112003263\&site=eds-live

34. Tabue Teguo M, Simo-Tabue N, Stoykova R, Meillon C, Cogne $\mathrm{M}$, Amiéva $\mathrm{H}$, et al. Feelings of Loneliness and Living Alone as Predictors of Mortality in the Elderly: The PAQUID Study. Psychosom Med [Internet]. 2016 [acceso mayo del 2017]; 78(8). Disponible en: http://journals.Iww.com/ psychosomaticmedicine/Fulltext/2016/10000/Feelings_of_ Loneliness_and_Living_Alone_as.4.aspx

35. Jung $H$, Lee J-E. The impact of community-based eHealth self-management intervention among elderly living alone with hypertension. J Telemed Telecare [Internet]. 2017 [acceso mayo del 2017];23(1):167-73. Disponible en: http://journals. sagepub.com/doi/10.1177/1357633X15621467

36. Stevens PJ, Syddall HE, Patel HP, Martin HJ, Cooper C, Aihie Sayer A. Is grip strength a good marker of physical performance among community-dwelling older people?. J Nutr Heal Aging. 2012;16(9):769-74.
37. Wu Y, Zhang D, Pang Z, Oksuzyan A, Jiang W, Wang S, et al. Gender-specific patterns in age-related decline in general health among Danish and Chinese: A cross-national comparative study. Geriatr Gerontol Int. 2012;12(3):431-9.

38. Van Houwelingen AH, Cameron ID, Gussekloo J, Putter $\mathrm{H}$, Kurrle S, De Craen AJM, et al. Disability transitions in the oldest old in the general population. The Leiden 85-plus study. Age (Omaha). 2014;36(1):483-93.

39. O’ Donnell ML VT, Holmes AC, Ellen S, Wade D, Creamer M el al. Disability after injury: the cumulative burden of physical and mental health. journals Clin psychiatry. 2013;74(2):13743.

40. Pirogovsky E, Martinez-Hannon M, Schiehser D, Lessig S, Song D, Litvan I, et al. Predictors of performance -based measures of instrumental activities of daily living in nondemented patients with Parkinson's disease. Journal of clinical and experimental neuropsychology. 2013;35(9):926-33.

41. Quach L, Yang FM, Berry SD, Newton E, Jones RN, Burr JA, et al. Depression, antidepressants, and falls among communitydwelling elderly people: The mobilize boston study. Journals Gerontol - Ser A Biol Sci Med Sci. 2013;68(12 A):1575-81.

42. Chao SF. Functional disability and depressive symptoms: longitudinal effects of activity restriction, perceived stress and social support. Aging Ment Health. 2014;18(6):767-76.

43. Barry LC, Soulos PR, Murphy TE, Kasl S, Gill TM. Association between indicators of disability burden and subsequent depression among older persons. Journals Gerontol - Ser A Biol Sci Med Sci. 2013;68(3):286-92.

Fuentes de financiamiento:

Este artículo ha sido financiado por sus autores.

Conflictos de interés:

Los autores declaran no tener ningún conflicto de interés.

\section{Correspondencia:}

Cristina Torres-Mallma

Dirección: Jr. Guardia Civil Norte 598 - Santiago de Surco

Teléfono: (01) 923 - 976 - 025

Correo electrónico: cristina_torres95@hotmail.com

Recibido: 03 de Julio de 2017 Evaluado: 05 de julio de 2017 Aprobado: 31 de Julio de 2017

(c) La revista. Publicado por Universidad de San Martín de Porres, Perú. (сc) ву Licencia de Creative Commons Artículo en acceso abierto bajo términos de Licencia Creative Commons Atribución 4.0 Internacional. (http: //creativecommons.org/licenses/by/4.0/) 